\title{
New form of administering chlorhexidine for treatment of denture-induced stomatitis
}

This article was published in the following Dove Press journal:

Therapeutics and Clinical Risk Management

13 June 201 I

Number of times this article has been viewed

\section{Soukaina Ryalat \\ Rula Darwish \\ Wala Amin}

Department of Oral Surgery, Jordan University, Amman, Jordan
Correspondence: Soukaina Ryalat PO Box 1669, Amman I1953, Jordan $\mathrm{Tel}+962795484463$

Fax +96265300248

Email salryalat@yahoo.com
Background: The purpose of this study was to evaluate the release of chlorhexidine as an antifungal drug from doped self-cured poly (methyl methacrylate) (PMMA) acrylic resin and the effect of the drug released on the growth of Candida albicans.

Methods: Release of chlorhexidine was evaluated using liquid chromatography, and the effect of the drug on the growth of $C$. albicans was investigated microbiologically using a "well" technique on Saboraud culture medium inoculated with a resistant strain of C. albicans.

Results: Chlorhexidine leached steadily out of the acrylic resin into distilled water at mouth temperature, and the sustained drug release continued throughout the 28 -day test period. The drug released also demonstrated antifungal activity against the resistant strain of C. albicans.

Conclusion: The findings of this study support the use of chlorhexidine-impregnated self-cured PMMA chair-side resin as a new dosage form for the treatment of denture-induced stomatitis.

Keywords: chlorhexidine, poly (methyl methacrylate), eluates, antifungal drug, denture stomatitis, Candida albicans

\section{Introduction}

Candida-induced denture stomatitis is a common form of oral candidosis that manifests as diffuse inflammation of the denture-bearing areas. Oral candidosis appears to be caused by a multiplicity of predisposing factors. ${ }^{1-3}$ Adherence of Candida albicans has been implicated as the first step in the pathogenesis of oral candidosis, ${ }^{4}$ and its relative cell hydrophobicity is also a contributory factor. ${ }^{5}$

Chlorhexidine gluconate is widely prescribed as an antiseptic mouthwash in dentistry due to its broad-spectrum antimicrobial activity, including $C$. albicans. ${ }^{6}$ The antifungal effect of chlorhexidine has been shown in many studies, and it has been demonstrated that exposure of $C$. albicans to chlorhexidine suppresses the ability of candida to adhere to buccal epithelial cells. Immersion of acrylic dentures in chlorhexidine suppresses adhesion of candida to the prosthesis. ${ }^{7}$ For these reasons, chlorhexidine may be considered as an appropriate alternative to conventional antimycotic drugs in the management of oral candidosis. ${ }^{8}$ It has been reported that the duration of inhibition of adherence of candida was longer-lasting when chlorhexidine was used rather than amphotericin $\mathrm{B}$ and nystatin. ${ }^{9}$

There are many oral delivery modes for chlorhexidine. It is used principally as a $0.2 \%$ mouthwash with a topical mode of action. However, most of the agent is removed from the oral cavity during the first hour due to the diluent effect of saliva and the cleansing effect of the oral musculature, possibly reducing its therapeutic efficacy. ${ }^{10}$ Biofilms of candida on mucosal and inert surfaces, such as dentures, may also contribute 
to therapeutic failure by modifying the susceptibility to antifungal agents. ${ }^{11}$ Poor patient compliance due to the need for frequent drug application and associated adverse effects could also result in recurrence of disease. ${ }^{12}$ A sustainedrelease delivery system for treatment of denture stomatitis using chlorhexidine incorporated into a tissue conditioner has been investigated, and it has been confirmed that there was a gradual release of the drug from the tissue conditioner and inhibition of candida growth in vitro. ${ }^{13}$ A topical sustainedrelease dosage form could help overcome the side effects of mouth rinsing with chlorhexidine and guarantee availability of the agent in the target area at a therapeutic dosage. Such a dosage form of chlorhexidine would be able to release the drug at a low therapeutic level over a long period of time, and might thus prevent tooth staining and the bitter taste. ${ }^{14}$ The emergence of chlorhexidine-resistant strains has not been observed clinically compared with the conventional antimycotic drugs, such as nystatin, amphotericin B, and recently mycostatin. ${ }^{15}$

Methods for detecting and estimating the eluates from poly (methyl methacrylate) (PMMA) acrylic denture bases have progressed over the past decades. The earlier methods were either physical or chemical in nature, ${ }^{16,17}$ but their poor detection limits rendered them unsuitable for detecting small amounts of leachate concentration. Therefore, these methods failed to provide an accurate estimation of leachates in acrylic resin bases. That was the situation until the advent of gas chromatography as a method of detecting residual monomers and other leachates in acrylic resin bases. ${ }^{18}$ The ease of this technique has made the procedure suitable for clinical application. The technique has been used in the detection of residual monomer and other leachates in whole saliva of human subjects wearing acrylic palatal appliances. ${ }^{19,20}$

More recently, a novel method of acquiring the test specimen for investigation by gas chromatography was introduced, ${ }^{21}$ whereby the eluates were extracted out of the polymerized acrylic resin plate using solvents. The resulting solution was then centrifuged, and the yield was subjected to gas chromatographic analysis for estimation of eluate concentrations. This method of investigation has been praised for its accuracy and precision, ${ }^{22,23}$ as well as detection sensitivity for extremely small amounts, ie, 1 ppm and less, of leachates.

The aim of the present investigation was to:

- Establish whether a chlorhexidine antifungal drug can be incorporated in a conventional autopolymerizing PMMA formulation

- Use the doped polymer for sustained intraoral delivery of chlorhexidine
- Investigate whether intraorally delivered chlorhexidine is of adequate concentration to have fungicidal activity against $C$. albicans and be a potential treatment for denture-induced oral candidiasis.

\section{Materials and methods Sample preparation}

A polymerized PMMA acrylic resin (Paladur ${ }^{\circledR}$, Heraeus Kulzer, Wehrheim, Germany) at room temperature was used in this study. The mixing ratio and conditions for processing and polymerization recommended by the manufacturer were followed strictly. The polymer powder/monomer liquid ratio for mixing was $5 \mathrm{~g} / 3 \mathrm{~mL}$. Two groups of disc-shaped PMMA specimens were prepared; samples for the first group were impregnated with chlorhexidine powder $10 \%$ $\mathrm{w} / \mathrm{w}$ and samples for the second group (controls) were not (Figure 1).

Incorporation of chlorhexidine $10 \% \mathrm{w} / \mathrm{w}$ within the polymerized PMMA denture base polymer was indicated by mechanical tests carried out in a pilot study on the polymer prior to the present investigation. The results of these tests indicated that chlorhexidine $10 \%$ was the maximum dose that could be safely incorporated in PMMA without having any undue effect on the mechanical properties of the polymer.

The antifungal drug was added in the specified ratio to the acrylic resin powder. The mixture of powders and liquid monomer was then stirred for 15 seconds and left standing for 4 minutes until a plastic dough was formed. The dough was then packed into a specially constructed disc-shaped steel mould to produce a disc specimen $(3.8 \mathrm{~mm}$ diameter and $1.0 \mathrm{~mm}$ thickness). After packing, the mould was allowed to stand for 13 minutes, then placed in a pressure curing unit and cured at $55^{\circ} \mathrm{C}$ and 2 bar pressure for 15 minutes.

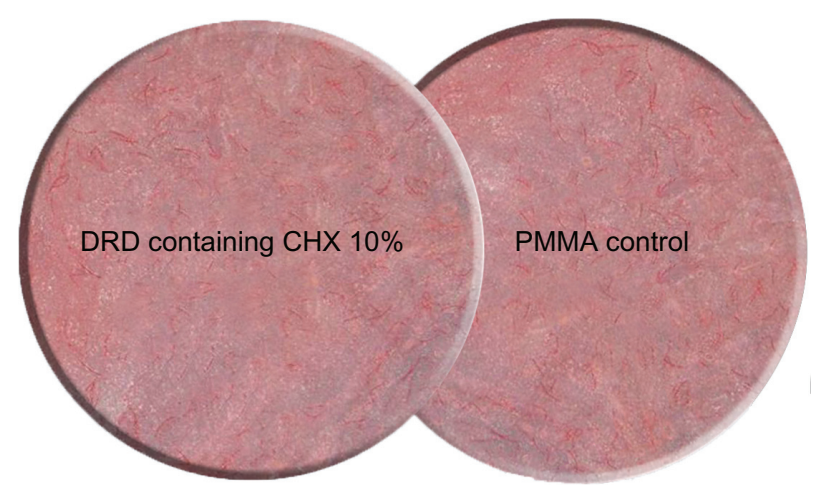

Figure I Discs of the two specimen groups showing the drug release device (DRD) impregnated with chlorhexidine (CHX) 10\% and the control poly (methyl methacrylate) (PMMA) disc. 


\section{High performance liquid chromatography}

Standard chlorhexidine was purchased from Cadilla Pharmaceuticals (Ahmad-Abad, India). The internal standard, p-methyl phenol, was purchased from Acros Organics (Geel, Belgium). The analysis was carried out on isocratic high-performance liquid chromatography apparatus consisting of the following components: a high-performance liquid chromatography pump (LC 1110; GBC Scientific Equipment, Melbourne, Australia), a high-performance liquid chromatography injector (Rheodyne 7125; PerkinElmer, Akron, OH), a high-performance liquid chromatography ultraviolet visible detector (LC 1205; GBC Scientific Equipment), and an integrator (LC 4290; Spectra Physics, Mountain View, CA). The high-performance liquid chromatography apparatus was operated under the following working conditions: acetonitrile/phosphate buffer $0.01 \mathrm{M}(25 \%: 75 \%)$ eluent, an eluent flow rate $1.0 \mathrm{~mL} / \mathrm{min}$, injection volume $20 \mu \mathrm{L}$, a BDSC18 column $(25 \mathrm{~cm} \times 4.6 \mathrm{~mm}$, particle size $5 \mu \mathrm{m})$, ultraviolet visible spectrophotometer ( $\lambda 210 \mathrm{~nm}$, range 1.0) detector, attenuation 8 , and integrator chart speed $0.5 \mathrm{~cm} /$ minute.

\section{Standard stock and working solutions}

A standard stock solution of $1000 \mu \mathrm{g} / \mathrm{mL}$ was prepared by dissolving $10 \mathrm{mg}$ of the drug in $10.0 \mathrm{~mL}$ of high-performance liquid chromatography water. The solutions prepared were kept in a refrigerator. The standard working solution of chlorhexidine was prepared by mixing $20 \mu \mathrm{g} / \mathrm{mL}$ of the drug with $50 \mu \mathrm{g} / \mathrm{mL}$ of the $p$-methyl phenol internal standard in high-performance liquid chromatography water.

\section{Leaching behavior}

In the first group of samples, five discs, weighing $6.32 \mathrm{~g}$ each and containing chlorhexidine $10 \% \mathrm{w} / \mathrm{w}$ were each placed separately in a $10 \mathrm{~mL}$ screw-capped vial and then covered with $5 \mathrm{~mL}$ of double-distilled water. The vials were left in a water bath at $37^{\circ} \mathrm{C}$ with continuous gentle shaking. After 1 hour, $150 \mu \mathrm{L}$ of aqueous solution of the corresponding internal standard $(160 \mathrm{nmol} / \mathrm{mL})$ was added and mixed. Finally, $20 \mu \mathrm{L}$ of this mixture were injected onto the high-performance liquid chromatography column according to the abovementioned conditions. This procedure was repeated at 24 hours, at days 2, 3, 4, 5, 6, and 7, and every week for the next 4 weeks. The same procedure was repeated for the five blank PMMA control specimens.

\section{Qualitative and quantitative analysis}

A representative chromatogram of a standard mixture of chlorhexidine and the internal standard is shown in Figure 2. The qualitative identification of the drug peak was performed
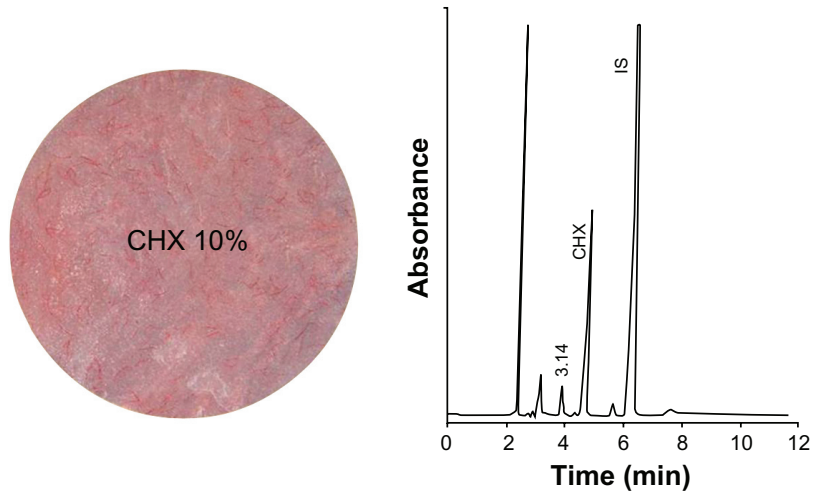

Figure 2 A representative chromatogram of a standard mixture of chlorhexidine with the internal standard.

Abbreviations: IS, internal standard; $\mathrm{CHX}$, chlorhexidine.

by comparing the relative retention time of the drug with respect to the internal standard in the real sample with that in the chromatogram for the standard drug mixture, which in this case was 4.860 minutes for chlorhexidine. The quantitative determination was performed using the relative peak areas and relative concentrations.

\section{Microbiological investigation}

A resistant strain of $C$. albicans was isolated from an inpatient at Jordan University Hospital and confirmed by biochemical tests. A reference strain of C. albicans (ATCC90028) was used as a control. Until testing, the yeasts were kept frozen in brain-heart broth (Difco Laboratories, Detroit, MI) with glycerol 5\%. For each experiment, the strains were subcultured twice on Saboraud agar (Difco Laboratories) for 24 hours at $35^{\circ} \mathrm{C}$ to ensure viability and purity. The inoculum suspension was prepared by picking five colonies of at least $1 \mathrm{~mm}$ in diameter and suspending them in $5 \mathrm{~mL}$ of sterile saline solution $0.85 \%$.

\section{Well diffusion test}

The inoculum used was prepared using yeasts from a 24-hour culture on Saboraud agar, with a suspension made in sterile saline solution $0.85 \%$. The turbidity of the suspension was adjusted using a spectrophotometer at $530 \mathrm{~nm}$ to obtain a final concentration matching that of a $0.5 \mathrm{McFarland}$ standard $\left(0.5-2.5 \times 10^{3}\right)$. The inoculated agar was poured into the assay plate $(9 \mathrm{~cm}$ in diameter) and allowed to cool down on a level surface. Once the medium had solidified, "wells" $4 \mathrm{~mm}$ in diameter were cut from the agar, and $20 \mu \mathrm{L}$ of the chlorhexidine $10 \%$ eluates were placed into a well in separate assay plates. Control cups containing the drug-release device only were also included in each assay plate. The plates were incubated at $35^{\circ} \mathrm{C}$ for 72 hours. 
The absence of growth of C. albicans, demonstrated by the occurrence of a growth inhibition zone around the wells containing the chlorhexidine-impregnated drug-release device was interpreted as antifungal drug activity, which was expressed by measurement of the diameter of the inhibition zone present around the well accommodating the drug-release device, using a PBI Readbiotic (PBI International, Milano, Italy). Each experiment was carried out five times for the antifungal drug-impregnated release device and controls.

\section{Results}

\section{Drug-release device}

At the outset, incorporation of chlorhexidine into the polymerized PMMA acrylic resin at room temperature did not have any untoward effect on the polymerization reaction of PMMA or on preparation of the test sample.

\section{Drug release}

The antifungal drug, ie, chlorhexidine $10 \%$, contained in the disc specimen demonstrated a high initial rate of elution from the PMMA drug-release reservoir during the first 2-7 days, followed by a controlled sustained elution process that continued throughout the 28-day test period (Table 1 and Figure 3). In general, chlorhexidine initially displayed a substantially high rate of release, reaching a concentration of up to $606 \mu \mathrm{g} / \mathrm{mL}$, which was equivalent to $36.4 \mathrm{mg}$ of drug released after 1 day. The rate of release thereafter became steadier until the end of the 28-day test period.

\section{Microbiology}

The percolate from the drug-release device containing chlorhexidine $10 \%$ showed clear antifungal activity. This was demonstrated by comparing the zones of inhibition of C. albicans growth around the wells containing the chlorhexidineimpregnated drug-release device with those containing the drug-release device alone (Figure 4). Chlorhexidine continued

Table I Mean concentration $(\mu \mathrm{g} / \mathrm{mL})$ and amount $(\mathrm{mg})$ values $(n=5)$ of the antifungal drugs released from the autopolymerized acrylic discs into distilled water at weekly intervals for the 28-day test period

\begin{tabular}{lll}
\hline Time (days) & \multicolumn{2}{c}{ Drug release device with chlorhexidine $\mathbf{1 0} \%$} \\
\cline { 2 - 3 } & Strength $(\mu \mathrm{g} / \mathbf{m L})$ & Amount $(\mathbf{m g})$ \\
\hline $\mathrm{I}$ & 606 & 36.4 \\
7 & 803 & 48.2 \\
14 & 885 & 53.1 \\
21 & 946 & 56.8 \\
28 & 974 & 58.4 \\
\hline
\end{tabular}

to demonstrate antifungal potential throughout the 28-day test period, as shown by the width of the inhibition zone of C. albicans growth vs time (Figure 5).

\section{Discussion}

The release of chlorhexidine from the PMMA drug-release device into distilled water indicates that polymerization of the PMMA acrylic resin did not adversely affect the antifungal drug nor did impregnation of the PMMA acrylic resin with chlorhexidine alter the diffusion characteristics of the resin. This finding is in agreement with that of previous studies using polymers for delivering chlorhexidine. ${ }^{24}$

The elution behavior of chlorhexidine from the autopolymerized acrylic resin showed relatively high initial release into distilled water during the first 4 days, during which the diffusion gradient of chlorhexidine was significantly higher than that demonstrated during the rest of the 28-day exposure to distilled water at mouth temperature.

The change in the rate of drug release is attributed to the fact that leaching of chlorhexidine into water is governed by a concentration-dependent diffusion process. ${ }^{25} \mathrm{~A}$ similar elution profile was reported for residual unpolymerized methyl methacrylate monomer from the same autopolymerizing acrylic resin when exposed to both distilled water and artificial saliva at mouth temperature. ${ }^{26,27}$

The results of the present investigation highlight the remarkable release behavior of an antifungal drug, chlorhexidine $10 \% \mathrm{w} / \mathrm{w}$, contained within a PMMA drug-release device. It has been clearly established that methacrylatebased polymers absorb up to $30 \%$ water depending on the osmolarity of the external solution ${ }^{16}$ and the formulation of a particular polymer. ${ }^{24,25}$ The mechanism of elution seemed to consist of two phases, ie, rapid linear behavior obeying Fick's law, followed by development of discrete clusters of the immersion liquid of unidentified osmotic activity. ${ }^{25}$

In the presence of chlorhexidine, the rapid elution phase indicates a surface release process. The subsequent slow sustained release may be the result of complex processes, involving formation of fluid clusters around the drug molecules and the interaction of these clusters with the mechanism of fluid absorption of the acrylic resin. Similar behavior has been reported for the release of another drug from a methacrylatebased polymeric system into distilled water. ${ }^{24}$ The elution behavior of chlorhexidine may also be enhanced by surface crazing and porosity, formed in the brittle PMMA by osmotic forces consequent to the inclusion of the antifungal drug. This is consistent with findings from a study using the same polymeric system for delivery of hydrocortisone..$^{28}$ 


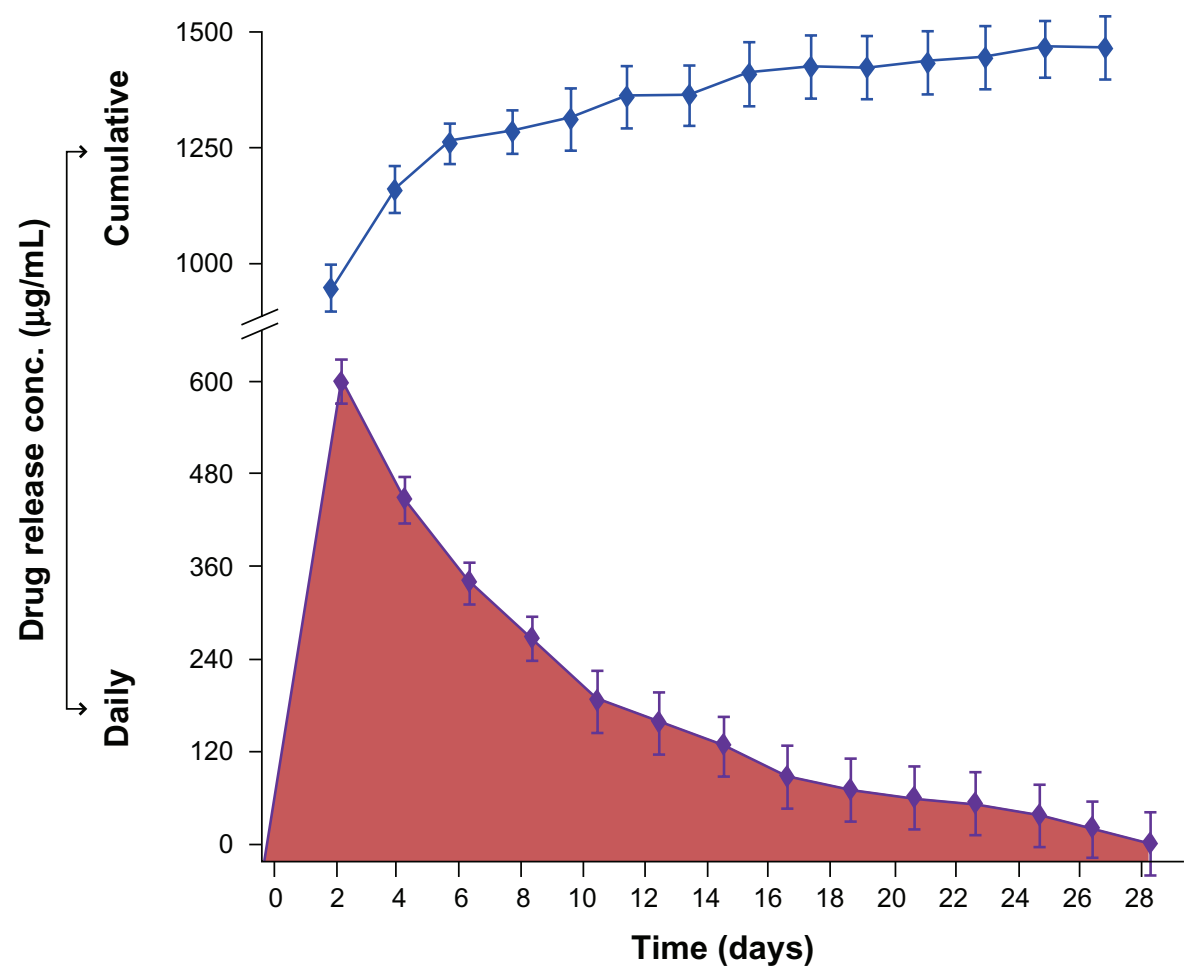

Figure $3 \mathrm{~A}$ plot of drug release concentration against time. The bottom part of the graph demonstrates the concentration of the released drug into distilled water daily. The immersion water was changed at every test interval. The top part of the graph demonstrates a hypothetical simulation of the clinical situation displaying what would be the cumulative concentration of chlorhexidine released at the denture/tissue interface and eventually absorbed by the tissues provided that the patient would wear the upper denture continually for 4 weeks. Error bars represent the distribution of data around the mean value of three repeats.

Having established that chlorhexidine-supplemented polymeric devices do release the antifungal drug in controlled concentrations for up to 4 weeks, it was essential to determine whether these concentrations were high enough to have antifungal activity against $C$. albicans, a common pathogen

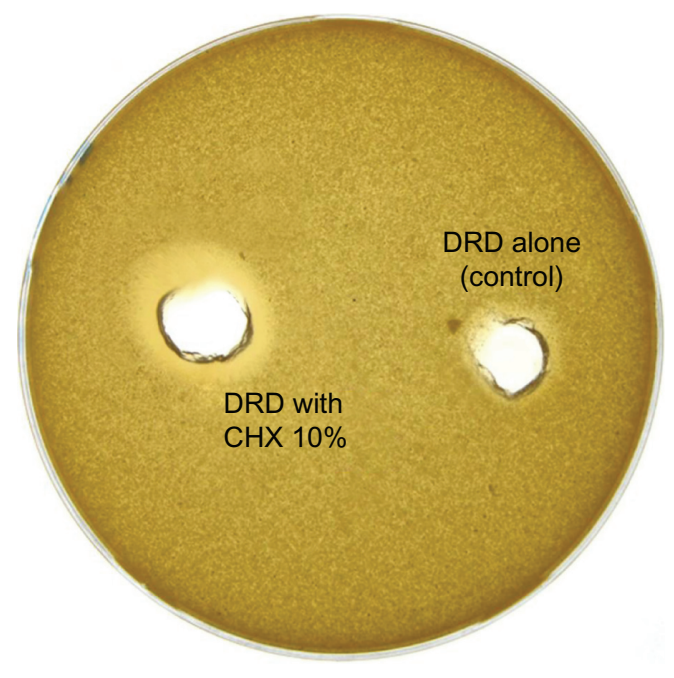

Figure 4 Zone of inhibition of Candida albicans growth around the well containing the drug release device (DRD) with 10\% chlorhexidine (CHX). Note the absence of an inhibition zone around the well containing the control, ie, poly (methyl methacrylate) alone. causing denture-induced stomatitis lesions. In this study, microbiological investigation showed that the antifungal drug was released at concentrations that did have an antifungal effect against $C$. albicans by inhibiting its growth in Saboraud culture for the 4-week test period. These findings

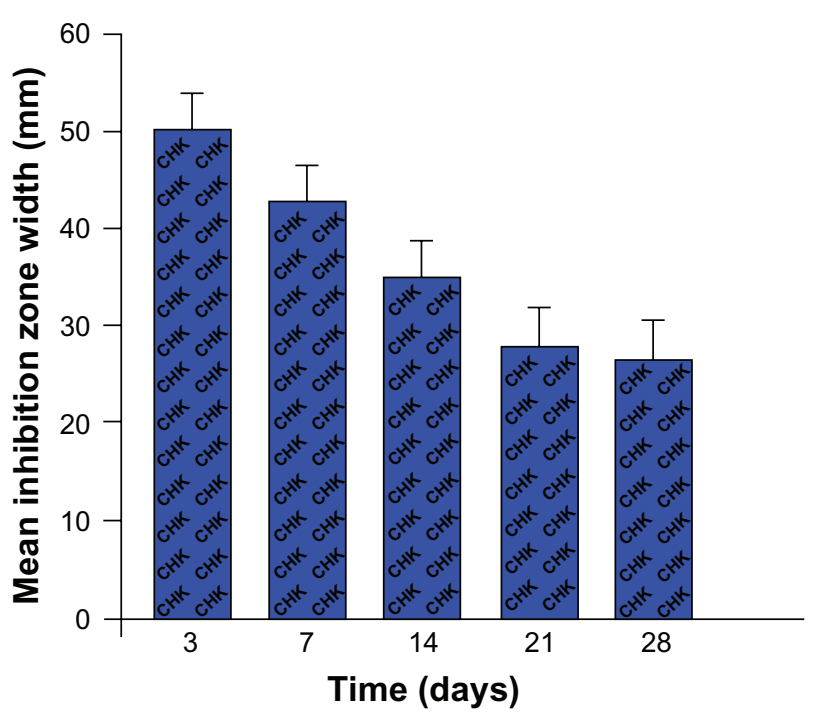

Figure 5 A histogram plotting the width of the inhibition zones of candidal growth affected by chlorhexidine over the 4-week test period. 
are consistent with those of an earlier study ${ }^{29}$ showing that chlorhexidine diffuses out of an autopolymerized acrylic resin in effective fungicidal concentrations over a period of at least 3 weeks.

Our results indicate that a chlorhexidine-supplemented drug-release device has a powerful antifungal effect, demonstrated by its capacity to inhibit growth of $C$. albicans. This interesting finding should encourage use of antifungal drugs in lower concentrations, thus reducing the chance of the host developing an allergic reaction to the drug, yet possessing a substantially high antifungal potential.

Future laboratory investigations could focus on studying the elution profile of chlorhexidine from an autopolymerized denture-based polymer into artificial saliva at different $\mathrm{pH}$ values. Such investigations may yield findings more comparable with the clinical situation. In the clinical context, the cumulative nature of chlorhexidine release into the surrounding fluid media helps saturate the salivary film, bathing the tissue surface of a denture base with continuous-release antifungal drug.

\section{Conclusion}

The findings of the present study show that the antifungal drug, chlorhexidine, can be successfully incorporated with autopolymerized PMMA, and that chlorhexidine leaches out of the polymer into the surrounding fluid medium in an environment similar to that in the oral cavity with respect to humidity and temperature. The sustained incremental elution of the antifungal drug from the impregnated-polymeric denture base maintains a gradually increasing concentration of the drug at the exact site where candidal infection lesions in the tissue bed of the maxillary denture are usually found. The results of the microbiological investigation in this study confirm the antifungal efficacy of the drug-supplemented delivery system and favor the use of polymeric systems doped with antifungal drugs in small concentrations. Clinical studies are essential in order to test the efficacy of the suggested drug delivery system, before implementing the new dosage form of antifungal drugs for the treatment of denture-associated oral candidiasis.

\section{Acknowledgments}

The authors express their sincere gratitude to Mrs Sana'a Alawi of the Department of Chemistry, Faculty of Sciences, University of Jordan, for her help in conducting the chromatography experiments. The authors would also like to express their thanks to Mr Ameed Al-Taher of the Faculty of Pharmacy, University of Jordan, for his invaluable help with the microbiology experiments.

\section{Disclosure}

The authors report no conflicts of interest in this work.

\section{References}

1. Samaranayake LP. Oral mycoses in HIV infection. Oral Surg Oral Med Oral Pathol. 1992;73(2):171-180.

2. Koray M, Ak G, Kurklu E, et al. Fluconazole and/or hexetidine for management of oral candidiasis associated with denture-induced stomatitis. Oral Dis. 2005;11(5):309-313.

3. Samaranayake LP. Host factors and oral candidosis. In: Samaranayake LP, MacFarlane TW, editors. Oral Candidosis. London, UK: Butterworth \& Company Ltd: 1990.

4. Ruechel R: Virulence factors of Candida species. In: Samaranayake LP, MacFarlane TW, editors. Oral Candidosis. London, UK: Butterworth \& Company Ltd: 1990.

5. Klotz SA, Drutz DJ, Zajic JE. Factors governing adherence of Candida species to plastic surfaces. Infect Immun. 1985;50(1):97-101.

6. MacNeill S, Rindler E, Walker A, Brown AR, Cobb CM. Effects of tetracycline hydrochloride and chlorhexidine gluconate on Candida albicans. An in vitro study. J Clin Periodontol. 1997;24(10):753-760.

7. Giuliana G, Pizzo G, Milici ME, Musotto GC, Giangreco R. In vitro antifungal properties of mouthrinses containing antimicrobial agents. J Periodontol. 1997;68(8):729-733.

8. Tobgi RS, Samaranayake LP, McFarlane TW. Adhesion of Candida albicans to buccal epithelial cells exposed to chlorhexidine gluconate. J Med Vet Mycol. 1987;25(5):335-338.

9. McCourtie J, McFarlane TW, Samaranayake LP. A comparison of the effects of chlorhexidine gluconate, amphotericin B and nystatin on the adherence of Candida species to denture acrylic. J Antimicrob Chemother. 1986;17(5):575-583.

10. Bonesvoll P, Lokken P, Rolla G. Retention of chlorhexidine in the human oral cavity after mouth rinses. Arch Oral Biol. 1974;19(11): 209-212.

11. Baillie GS, Douglas LJ. Candida biofilms and their susceptibility to antifungal agents. Methods Enzymol. 1999;310:644-656.

12. Budtz-Jorgensen E. Etiology, pathogenesis, therapy, and prophylaxis of oral yeast infections. Acta Odontol Scand. 1990;48(1):61-69.

13. Addy M, Thaw M. In vitro studies into the release of chlorhexidine acetate, prednisolone sodium phosphate, and prednisolone alcohol from cold cure denture base acrylic. J Biomed Mater Res. 1982;16(2):145-157.

14. Friedman M, Golomb G. New sustained release dosage form of chlorhexidine for dental use. J Perio Res. 1982;17(3):323-328.

15. Thurmond JM, Brown AT, Sims RE, et al. Oral Candida albicans in bone marrow transplant patients given chlorhexidine rinses: Occurrences and susceptibilities to the agent. Oral Surg Oral Med Oral Pathol. 1991;72(3):291-295.

16. Wolcott RB, Paffenbarger GC, Schoonover IR. Direct resinous filling materials: Temperature rise during polymerization. J Am Dent Assoc. 1951;42(5):253-263.

17. Smith DC, Bains MED. The detection and estimation of residual monomer in polymethyl methacrylate. J Dent Res. 1956;35(1):16-24.

18. Caul HJ, Sweeney WI, Paffenbarger GC. Relationship between residual monomer and some properties of self-curing dental resins. J Am Dent Assoc. 1956;53(1):60-63.

19. Anderson JN. Applied Dental Materials. 5th ed. Oxford, UK: Blackwell; 1976.

20. Austin AI, Basker RM. The level of residual monomer in acrylic denture base materials. Br Dent J. 1980;149(10):281-286.

21. Sadamori S, Kotani H, Hamada T. The usage period of dentures and their residual monomer content. J Prosthet Dent. 1992;68(2):374-376.

22. Urban VM, Cass QB, Oliveira RV, Giampaolo ET, Machado AL. Development and application of methods for determination of residual monomer in dental acrylic resins using high performance liquid chromatography. Biomed Chromatogr. 2006;20(4):369-376. 
23. Chai XS, Hou QX, Schork FJ. Determination of residual monomer in polymer latex by full evaporation headspace gas chromatography. J Chromatogr A. 2004;1040(2):163-167.

24. Riggs PD, Braden M, Patel M. Chlorhexidine release from room temperature polymerizing methacrylate systems. Biomaterials. 2000;21(4): 345-351.

25. Patel MP, Cruchley AT, Coleman DC, Swai H, Braden M, Williams DM. A polymeric system for intra-oral delivery of an anti fungal agent. Biomaterials. 2001;22(17):2319-2324.

26. Amin WM. A study of adhesion between soft lining materials and poly (methyl methacrylate). PhD Thesis, University of London, UK, 1987.
27. Alawi MA, Amin WM. Effect of aging on monomer elution from poly (methyl methacrylate) resin under simulated intra-oral conditions. FEB. 2007;16(4):408-414.

28. Brook IM, van Noort R. Drug release from acrylic polymers via channels and cracks: In vitro studies with hydrocortisone. Biomaterials. 1985;6(4):281-285.

29. Lamb DJ, Martin M. An in vitro and in vivo study of the effect of incorporation of chlorhexidine into autopolymerizing acrylic resin plates upon the growth of Candida albicans. Biomaterials. 1983;4(3):205-209.

\section{Publish your work in this journal}

Therapeutics and Clinical Risk Management is an international, peerreviewed journal of clinical therapeutics and risk management, focusing on concise rapid reporting of clinical studies in all therapeutic areas, outcomes, safety, and programs for the effective, safe, and sustained use of medicines. This journal is indexed on PubMed Central, CAS,
EMBase, Scopus and the Elsevier Bibliographic databases. The manuscript management system is completely online and includes a very quick and fair peer-review system, which is all easy to use. Visit http://www.dovepress.com/testimonials.php to read real quotes from published authors.

Submit your manuscript here: http://www.dovepress.com/therapeutics-and-clinical-risk-management-journal 